\title{
Influence of Electric Field on Electronic States of Graphene Nanoribbons under FET Structure
}

\author{
Ayaka Yamanaka* and Susumu Okada \\ Graduate School of Pure and Applied Sciences, University of Tsukuba, 1-1-1 Tennodai, Tsukuba, Ibaraki \\ 305-8571, Japan
}

\begin{abstract}
We study the electronic properties of graphene nanoribbons with zigzag and armchair edges under a parallel electric field by two planer electrodes with a potential barrier simulating an insulating layer of electrodes in FET structure using density functional theory combined with an effective screening medium method. Our calculations show that the nearly free electron (NFE) states strongly depend on the mutual arrangements of graphene nanoribbons with respect to the electric field. In contrast, the electronic energy bands associated with the $\pi$ electrons are insensitive to the relative direction of the ribbon with respect to the external electric field. We also observe that the electric field concentration around the edges leads to the orientation dependence of the NFE states on the field.
\end{abstract}

\section{Introduction}

The surfaces of matter disrupt the translational symmetry of crystals, leading to localized states around the surfaces. At the clean surfaces of materials with covalent networks, unsaturated covalent bonds at the surface atoms result in dangling bond states near the Fermi level.,2) These states affect the surface atomic arrangements and electronic structures, resulting in an interesting variation of the surface atomic morphologies. ${ }^{3-7)}$ In addition to the surface localized states, it has been reported that peculiar surface states are observed on the metal and semiconductor surfaces because of the quantum spill of electrons outside the atomic networks of these materials that act as an attracting potential for the states distributed in the vacuum region. The states exhibit a floating nature in the vacuum region and extend throughout the surfaces with free electron nature. ${ }^{8-11)}$ For layered materials, such as graphite, ${ }^{12,13)}$ hexagonal boron nitride (h-BN), ${ }^{14)}$ and transition metal dichalcogenides (TMDCs), ${ }^{15,16)}$ owing to their two-dimensional network of fully saturated bonds with atom thickness, the unusual surface states are distributed above and below the atomic networks and extend along the

\footnotetext{
${ }^{*}$ E-mail address: ayamanaka@comas.frsc.tsukuba.ac.jp
} 
layers in the conduction bands with a quadric dispersion. ${ }^{17-21)}$ In this case, the states are known to be nearly free electron (NFE) states or inter layer bands, which play decisive roles on the excited state spectrum and also on the ground state electronic properties upon the intercalation of foreign atoms or molecules into the spacing. ${ }^{22)}$ According to the unoccupied and extended nature of NFE states, NFE states are tunable by applying an external electric field normal to the atomic layers of these layered materials. ${ }^{21,23,24)}$ NFE states shift to lower energies under an external electric field and cross the Fermi level at a certain critical electric field that strongly depends on the thickness of the layered materials and the surface morphologies. These downward shifts of the NFE states by the perpendicular electric field are ascribed to the potential gradient outside the films by the electric field that effectively leads to an attractive potential for the electronic states.

In addition to two-dimensional surfaces, NFE states are also observed outside the edges of the graphene nanoribbons, which can be regarded as the one-dimensional version of the surfaces. ${ }^{25,26)}$ Furthermore, the edge NFE states also shift to lower energies with the injection of electrons or by applying the parallel electric field, and cross the Fermi level above the critical electron density or field. The state is primarily distributed at the vacuum region dislodged from the leftmost edge atomic site and is also slightly distributed at the atomic site near the edge atomic site, while, in contrast, the state is extended along the direction parallel to the ribbon with almost uniform distribution in the vacuum region. This fact implies that the NFE states exhibit a diverse dependence of their distribution on the relative arrangement of the ribbon to the external electric field.

In this work, we investigate the influence of the electric field direction on the NFE states of graphene nanoribbons using the first principles total energy calculations based on density functional theory combined with an effective screening medium method. Our calculations suggest that the NFE states strongly depend on the mutual arrangements of graphene nanoribbons with respect to the electric field. The NFE states distributed outside the edge depend sensitively on the lateral electric field, while the states weakly depend on the normal electric field. In contrast, the NFE states above and below the ribbons weakly depend on the electric field irrespective of the field directions. Electric field concentration around the ribbon edges occurs under the parallel field, leading to the difference in the sensitivity of the NFE states to the field. 


\section{Methods and Models}

The electronic structures of graphene nanoribbons under an electric field were investigated by the first-principles total energy calculations based on the density functional theory $^{27,28)}$ combined with an effective screening medium method ${ }^{29)}$ using the Simulation Tool for Atom TEchnology (STATE). ${ }^{30)}$ We used the local density approximation to treat the exchange-correlation interactions among the interacting electrons using Perdew-Zunger functional form fitting to the Ceperley-Alder numerical result. ${ }^{31,32)}$ Ultrasoft pseudopotentials were adopted to describe the electron-ion interactions generated by the Vanderbilt scheme. ${ }^{33)}$ The valence wave function and charge density were expanded in terms of the plane wave basis set with cutoff energies of 25 and $225 \mathrm{Ry}$, respectively.

In the present work, we consider zigzag and armchair graphene nanoribbons whose edge atomic sites are fully terminated by $\mathrm{H}$ atoms. To simulate the behavior of the graphene nanoribbons in a parallel electric field, we adopted the effective screening medium method to solve the Poisson equation under the boundary conditions including the external parallel electric field. We applied an electric field between two parallel metal electrodes, simulated by an effective screening medium, which are situated alongside the rightmost and the leftmost edges of the nanoribbons with $d=6 \AA$ vacuum regions (Fig. 1). In this case, the anode and cathode electrodes were set to the left side $(z=$ $-c / 2)$ and the right side $(z=c / 2)$ of the unit cell, as shown in Fig. 1, where $c$ is the lattice constant along the $\mathrm{z}$ direction. We set the potential barrier with the hight of 6.0 $\mathrm{eV}$ at $3.5 \AA$ from the rightmost edges of the nanoribbons to prevent the unintentional electron spill under the strong electric field. To investigate the electronic structures of the graphene nanoribbons with respect to the relative direction to the electric field, we rotate the nanoribbons around the ribbon axis with angles between $0^{\circ}$ and $90^{\circ}$. The atomic structures of the nanoribbons were fully optimized under a zero electric field until the force acting on each atom was less than $5 \mathrm{mRy} / \AA$. Integration over the Brillouin zone was carried out using equidistant $k$-point sampling in which 4 and 6 $k$-points were taken in the ribbon direction for the armchair and the zigzag ribbons, respectively. During the calculations under the condition of a finite electric field up to $0.5 \mathrm{~V} / \AA$, the atomic positions were fixed to those under the condition of a zero electric field. 


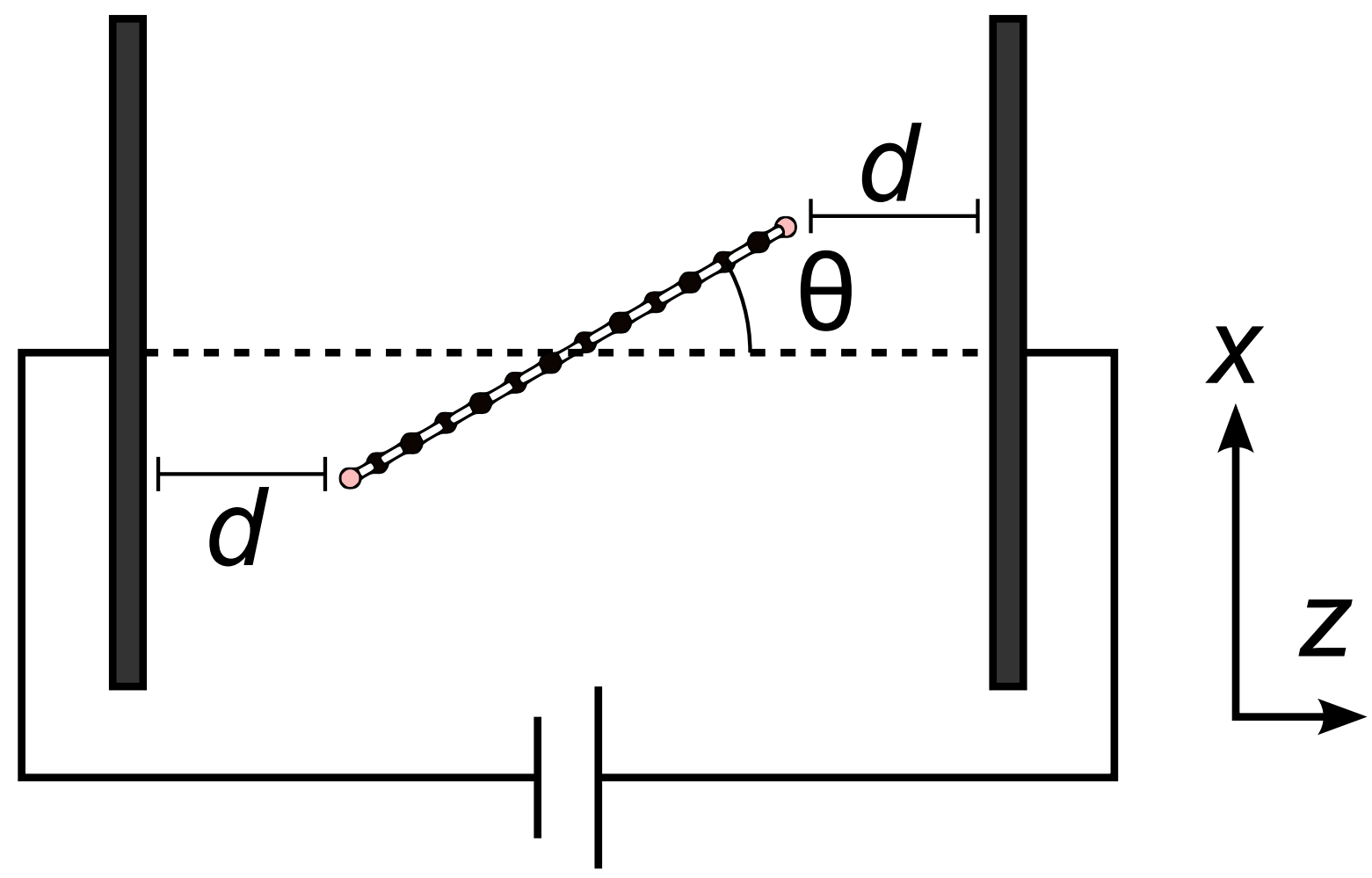

Fig. 1. Structural model of graphene nanoribbons under an electric field. Dark shaded rectangles represent metal electrodes simulated by the effective screening medium method.

\section{Results and Discussion}

Figure 2 shows the electronic energy band of graphene nanoribbons under zero and 0.5 $\mathrm{V} / \AA$ electric fields. For both zigzag and armchair ribbons, the NFE states shift to lower energies for all rotation angles under the external electric field. The downward shift of the NFE states strongly depends on the rotation angle $\theta$. For $\theta=0^{\circ}$, the lowest branch of the NFE states is shifted by 2.84 and $3.44 \mathrm{eV}$ compared with those under zero field for zigzag and armchair ribbons, respectively. In contrast, shifts from the zero-field values are 0.36 and $0.22 \mathrm{eV}$ for zigzag and armchair ribbons, respectively, with the rotation angle of $\theta=90^{\circ}$.

Figure 3 shows the contour plots of the squared wave function of NFE states above the Fermi level $\left(E_{F}\right)$ under a parallel electric field of $0.5 \mathrm{~V} / \AA$. For the ribbons with a rotation angle of $\theta=0^{\circ}$, the lowest unoccupied (LU) state has NFE nature with the maximum distribution existing outside the graphene edge and also extending along the edge in both the zigzag and armchair ribbons. By focusing on the higher branches of the quadric dispersion bands above the $E_{F}$ at the $\Gamma$ point, we found that the LU+2 in the zigzag ribbon and $\mathrm{LU}+3$ in the armchair ribbon exhibit a NFE state nature, which 
(a)

$$
\text { E.F. }=0 \mathrm{~V} / \mathrm{A} \quad \text { E.F. }=0.5 \mathrm{~V} / \mathrm{A}
$$
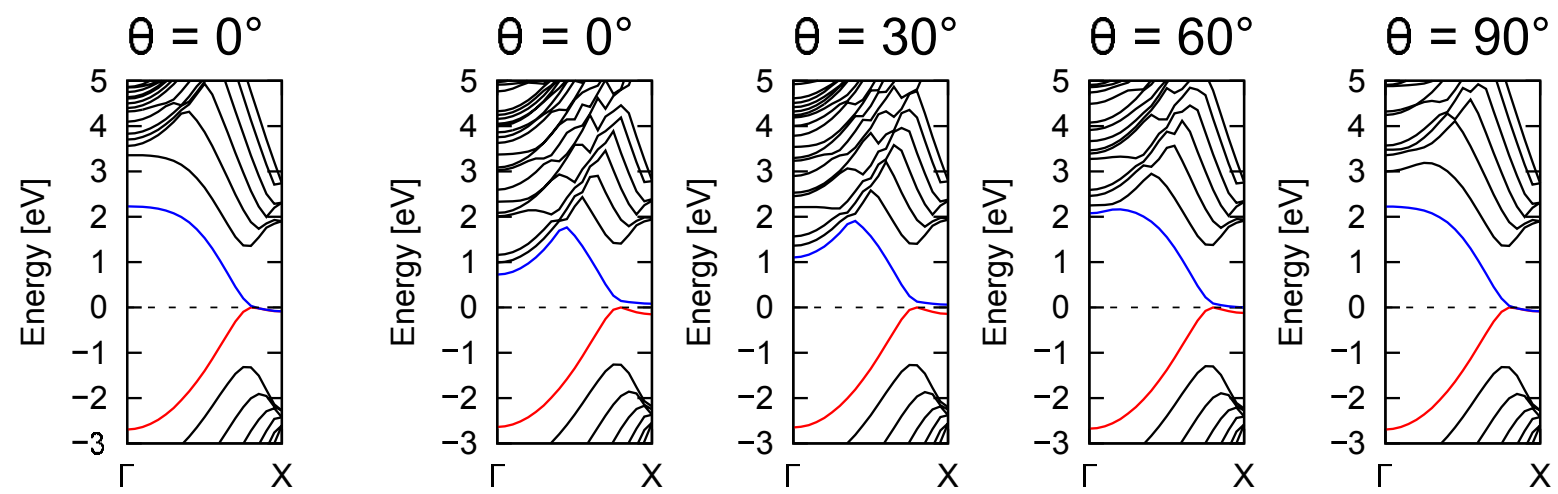

(b)
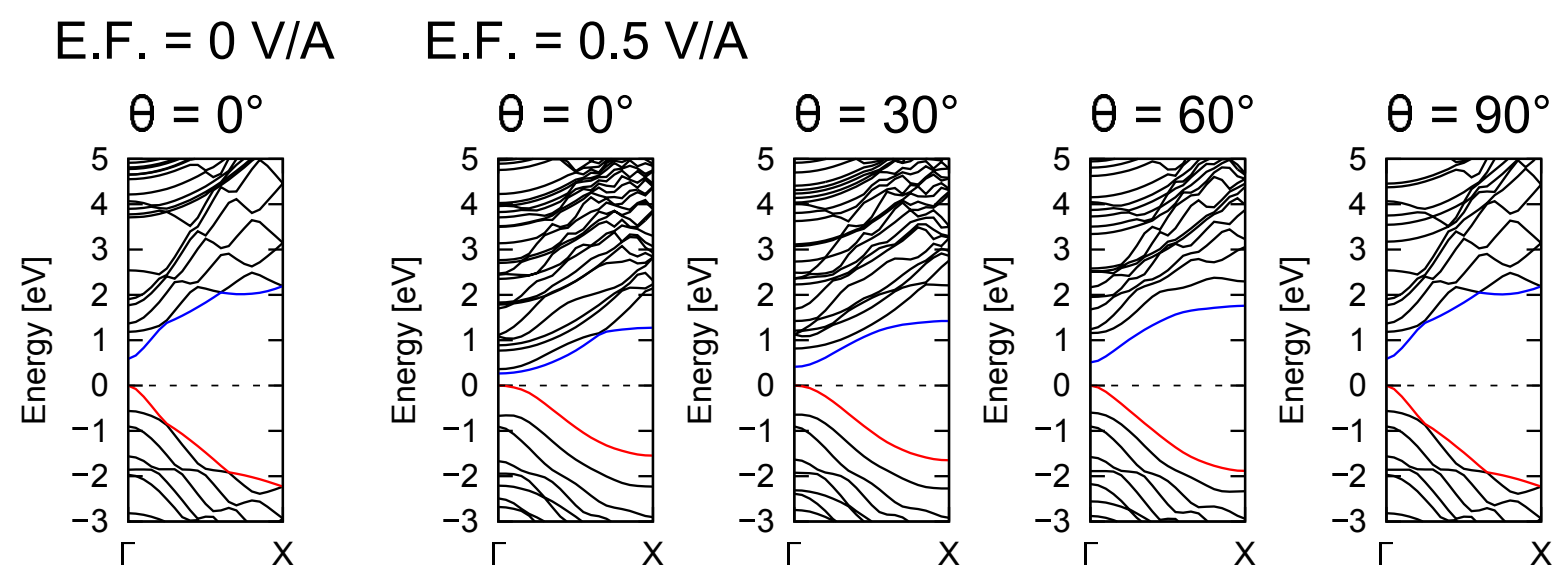

Fig. 2. Electronic energy band of (a) zigzag and (b) armchair graphene nanoribbons under zero and $0.5 \mathrm{~V} / \AA$ electric field. The energies are measured from that of the Fermi level.

is also distributed outside the rightmost atomic sites with a node with respect to the ribbon layer.

By rotating the ribbon, the NFE states exhibit a hybrid nature with the $\pi, \sigma$, and other NFE states for the rotation angle of $60^{\circ}$. The LU state is mainly distributed in the vacuum region outside the zigzag ribbon along with a small distribution on the $\pi$ states [Fig. 3(a): LU state under $\theta=60^{\circ}$ ]. A similar hybrid nature on the localized states of the graphene ribbons is also observed in the $\mathrm{LU}+3$ in the zigzag ribbon and the $\mathrm{LU}+4$ in the armchair ribbon. The $\mathrm{LU}+3$ in the zigzag ribbon has the hybridized nature between the NFE states alongside the edge and above and below ribbon [Fig. 3(a): LU+3 state under $\theta=60^{\circ}$. On the other hand, NFE state is hybridized with $\sigma$ states in the $\mathrm{LU}+4$ in armchair ribbon [Fig. $3(\mathrm{~b})$ : $\mathrm{LU}+4$ state under $\theta=60^{\circ}$ ]. 
For the zigzag ribbons with a rotation angle of $\theta=90^{\circ}$, we can observe two different distributions of the NFE states: The lowest branch of the NFE is distributed outside both edges of the ribbon while the next lowest branch of the NFE states is distributed above the ribbon, as observed for the conventional NFE states of bulk graphene. For these NFE states, the external electric field dislodges their distributions to the electrode side. In sharp contrast, in the case of an armchair ribbon with a rotation angle of $90^{\circ}$, no NFE states are observed in the lower conduction bands. These results indicate that the distribution of NFE states strongly depends on the mutual arrangement of the graphene nanoribbons with respect to the electric field.

Next, we investigate how the NFE states of the graphene nanoribbons depend on the electric field strength. Figure 4 shows the NFE position of the zigzag nanoribbon as a function of the parallel electric field. For the zigzag ribbon with a rotation angle of $\theta=0^{\circ}$, the NFE state distributed at the ribbon side rapidly shifts to lower energy with an increasing electric field, while the NFE states distributed above the plane gradually shift downward with an increasing electric field. For the ribbon with an angle of $\theta=90^{\circ}$ or with a perpendicular field, both NFE states weakly depend on the external field. The magnitude of the downward shifts is a tenth smaller than that for the side NFE state under a parallel field. Thus, the NFE state at the ribbon side depends sensitively on the parallel electric field. It should be noted that the NFE states rapidly shift downward by removing the potential barriers at the right side of the cell, because of the increase of vacuum region that decrease the kinetic energy of the states.

To elucidate the physical origin of the influence of the field direction on the NFE states, we investigated the electrostatic potential on the ribbons as well as the electric field evaluated by taking the gradient of the potential. Fig. 5 shows the contour and vector plots of the electrostatic potential and the calculated electric field, respectively. For the zigzag ribbons with a rotation angle of $\theta=0^{\circ}$, the electric field is highly concentrated around the graphene edge. By increasing $\theta$, the field concentration decreases, and the ribbon finally does not modulate the external field for a rotation angle of $\theta=90^{\circ}$. By comparing Fig. 4 with Fig. 5, we observed that the distribution of the NFE states is qualitatively the same as the regions where an electric field concentration occurs. The electric field concentration causes a substantial electrostatic potential gradient around the edge atomic sites, leading to the downward shift of NFE states alongside the edge. Thus, the electric field concentration is the physical origin of the sensitivity of NFE states with respect to the field direction and strength. 
Our results indicate that the graphene nanoribbons could provide the onedimensional free electron carriers in vacuum space alongside the ribbon under the lateral electric field. Since the NFE carriers are free from the scatters such as atomic vacancies and adatom on atomic network, the carrier are expected to be moderate mobility with remarkable stability. Furthermore, the emergence of the NFE states near the Fermi level under the electric field also give the theoretical insight into the field emission images from the edge of graphene nano ribbons.

\section{Summary}

We investigated the electronic structures of hydrogenated graphene nanoribbons under an electric field in terms of the field direction and strength, by performing the firstprinciple total energy calculations based on the density functional theory combined with the effective screening medium method. We found that the NFE states strongly depend on the mutual arrangements of graphene nanoribbons with respect to the electric field. The NFE states distributed outside the edge depend sensitively on the lateral electric field, while the states weakly depend on the normal electric field. In contrast, the NFE states above and below the ribbons weakly depend on the electric field irrespective of the field directions. Detailed analysis on the electrostatic potential of the graphene ribbons under an electric field clarified that the physical origin of the field direction dependence is the electric field concentration around the ribbon edges as in the classical electrodynamics. The field concentration around the edges enhances the gradient of electrostatic potential around the edge atomic sites, leading to a substantial downward shift of NFE states with an increasing electric field.

\section{5. acknowledgements}

AY acknowledges the Grant-in-Aid for JSPS Fellows. This work was partially supported by CREST, the Japan Science and Technology Agency, and a Grant-in-Aid for Scientific Research from the Ministry of Education, Culture, Sports, Science and Technology of Japan. 
(a)
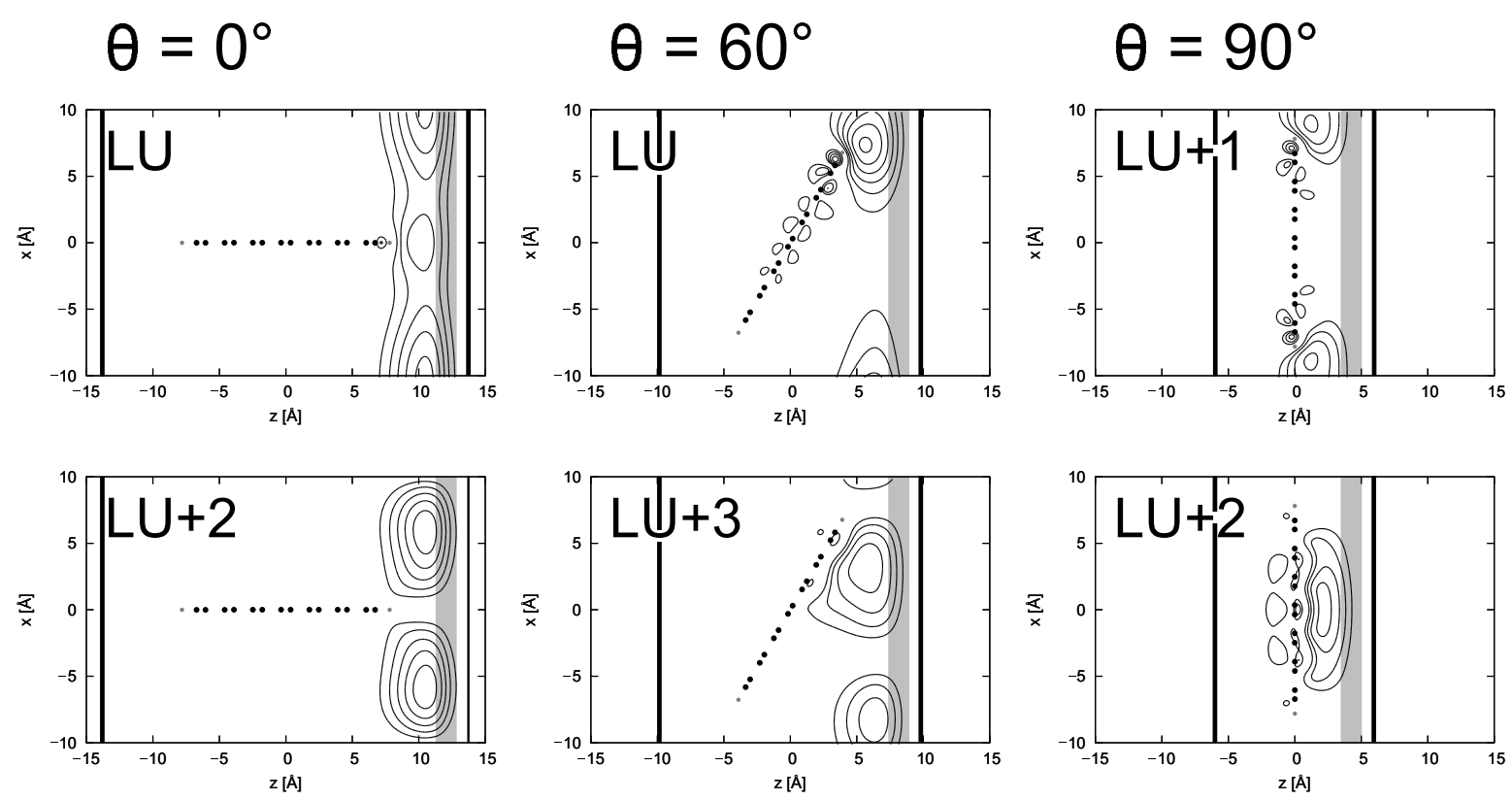

(b)
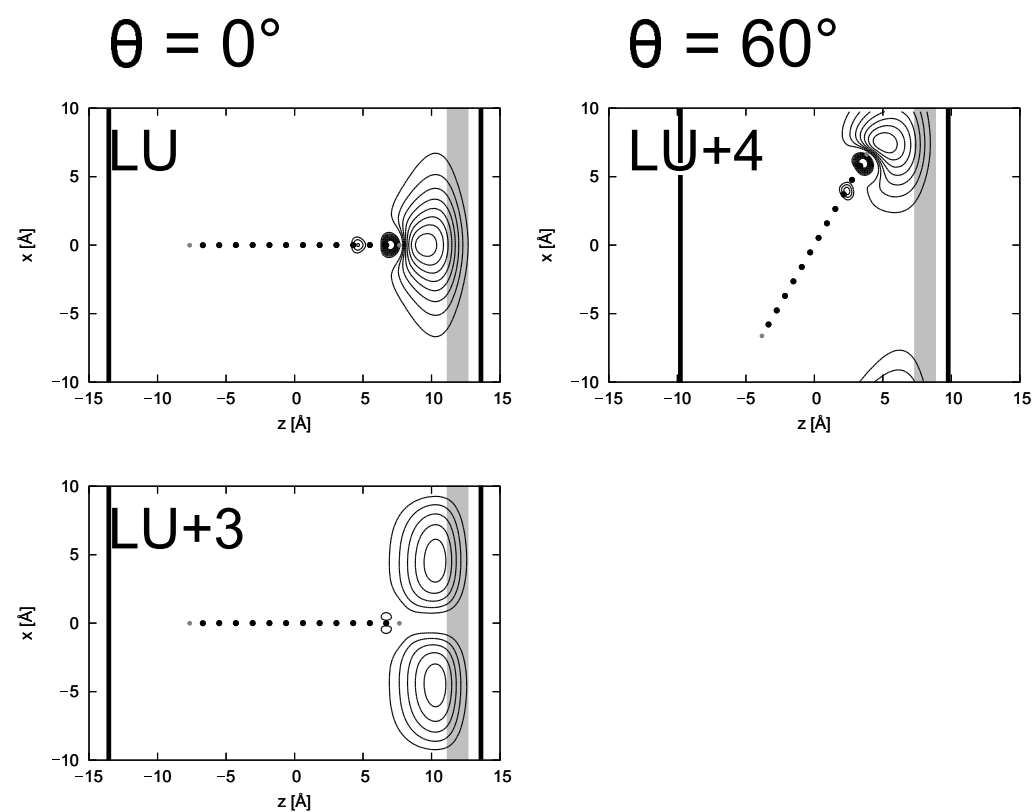

Fig. 3. Side views of contour plots of the NFE states of (a) zigzag and (b) armchair nanoribbons under a $0.5 \mathrm{~V} / \AA$ electric field. Black and gray circles denote the atomic positions of carbon and hydrogen, respectively. Gray rectangles denote the potential barriers to prevent unintentional electron spill. Thick black lines in left and right sides of the cell denote the positions of electrodes. 

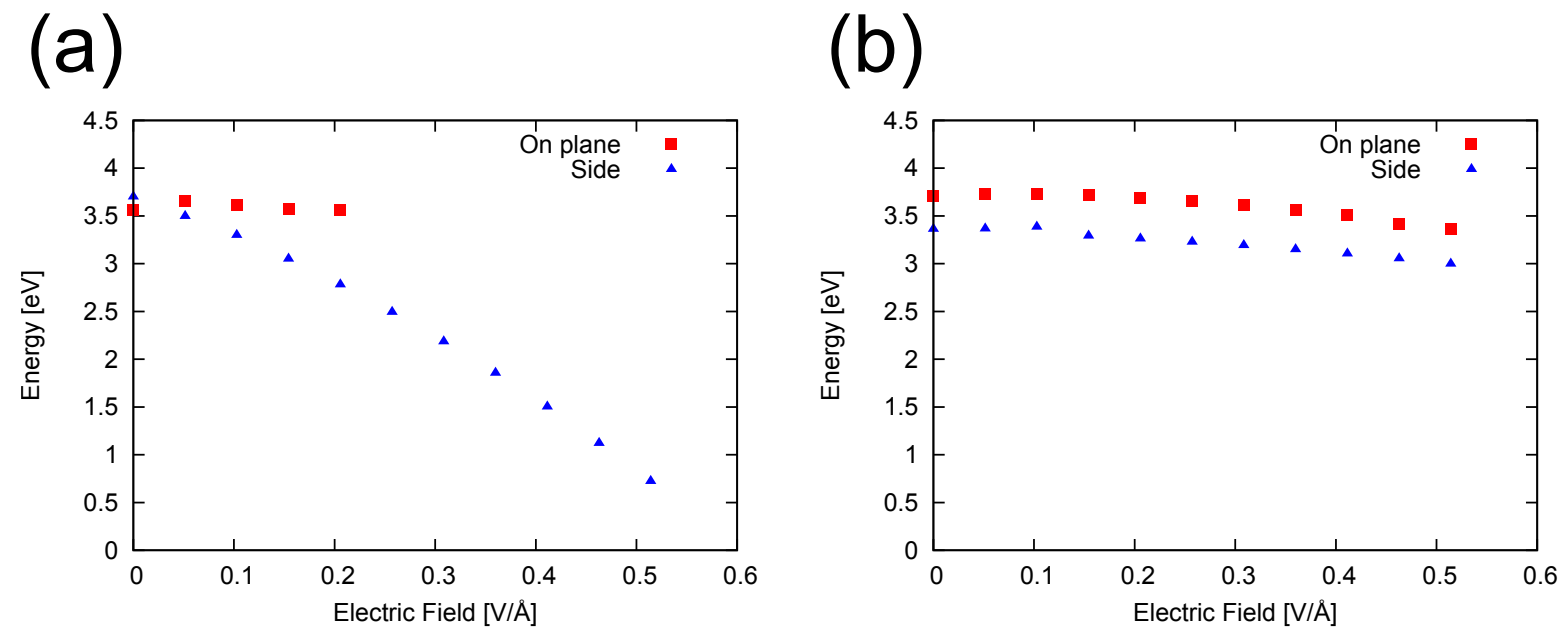

Fig. 4. NFE positions for the zigzag ribbon where rotation angles $\theta$ are (a) $0^{\circ}$ and (b) $90^{\circ}$ as a function of the electric field. The energies are measured from that of the Fermi level. Triangles and squares denote the NFE states that are distributed beside and on the plane of the ribbons, respectively. 
(a)

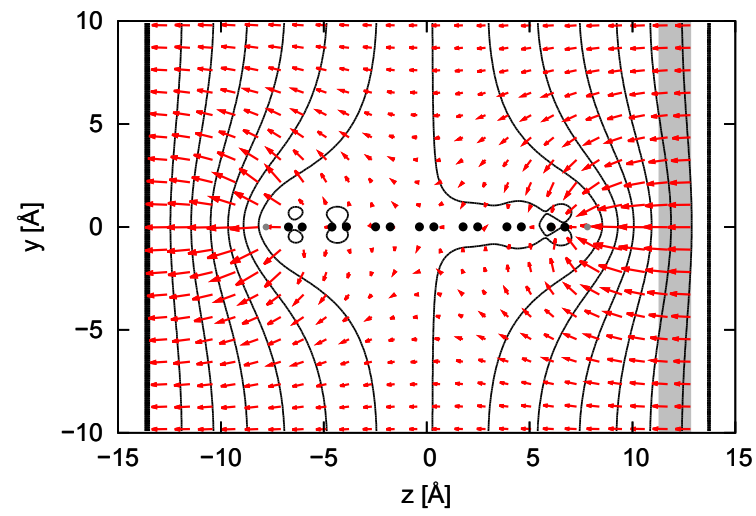

(c)

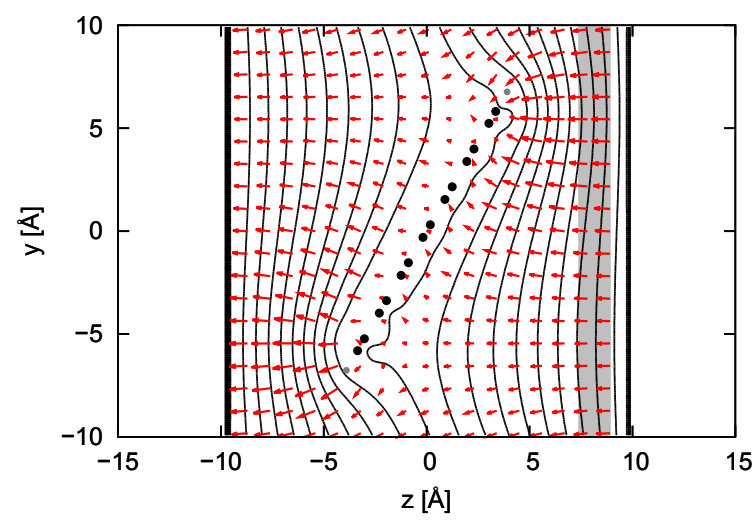

(b)

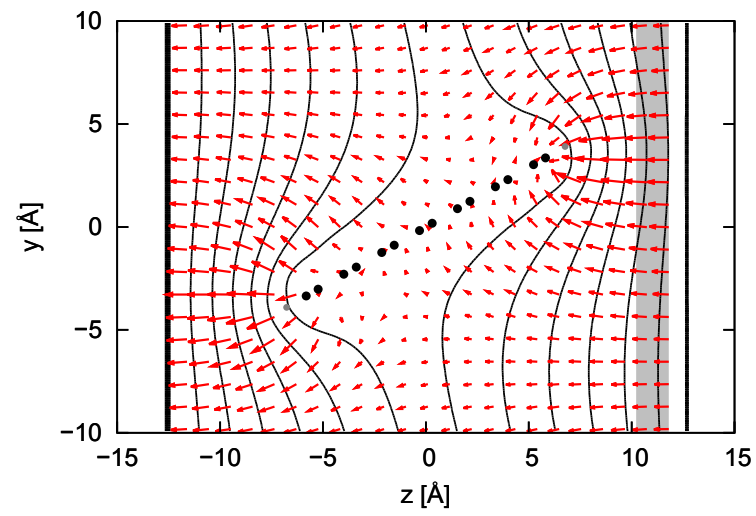

(d)

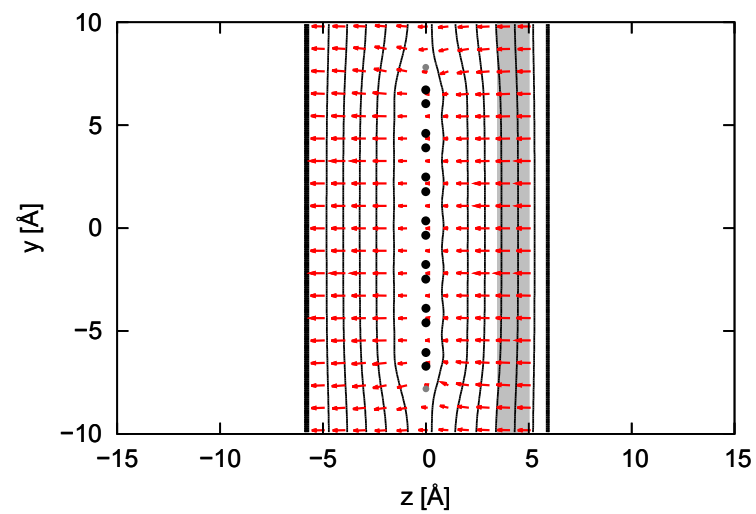

Fig. 5. Contour and vector plots of the electrostatic potential and electric field, respectively, of zigzag ribbons with rotation angles $\theta$ of (a) $0^{\circ}$, (b) $30^{\circ}$, (c) $60^{\circ}$, and (d) $90^{\circ}$ under a $0.5 \mathrm{~V} / \AA$ electric field. Black and gray circles denote carbon and hydrogen atomic positions, respectively. Gray rectangles denote the potential barriers to prevent unintentional electron spill. 


\section{References}

1) B.P. Lemke and D. Haneman, Phys. Rev. B 17, 1893 (1978)

2) W. Shockley, Phys. Rev. 56, 317 (1939)

3) J.J. Boland, Adv. Phys. 42, 129 (1993).

4) K. Oura, V.G. Lifshits, A.A. Saranin, A.V. Zotov, and M. Katayama, Surf. Sci. Rep. 35, 1 (1999).

5) D. Haneman, Phys. Rev. 121, 1093 (1961).

6) K.C. Pandy, Phys. Rev. Lett. 47, 1913 (1981).

7) K. Takayanagi, Y. Tanishiro, M. Takahashi, and S. Takahashi, J. Vac. Sci. Technol. A 3, 1502 (1985).

8) H. Conrad, G. Ertl, J. Kuppers, and E.E. Latta, Surf. Sci. 58, 578 (1976).

9) P. Heimann, H. Neddermeyer, and H.F. Roloff, J. Phys. C 10, L17 (1977).

10) S.G. Louie, Phys. Rev. Lett. 40, 1525 (1978).

11) S. LaShell, B.A. McDougall, and E. Jensen, Phys. Rev. Lett. 77, 3419 (1996).

12) M.S. Dresselhaus and G. Dresselhaus, Adv. Phys. 30, 139 (1981).

13) A.H. Castro Neto, F. Guinea, N.M.R. Peres, K.S. Novoselov, and A.K. Geim, Rev. Mod. Phys. 81, 109 (2009).

14) D. Golberg, Y. Bando, Y. Huang, T. Terao, M. Mitome, C. Tang, and C. Zhi, ACS NANO 6, 2979 (2010).

15) M. Chhowalla, H.S. Shin, G. Eda, L.-J. Li, K.P. Loh, and H. Zhang, Nature Chem. 5, 263 (2013).

16) Q.H. Wang, K. Kalantar-Zadeh, A. Kis, J.N. Coleman, and M.S. Strano, Nature Nanotech. 7, 699 (2012).

17) M. Posternak, A. Baldereschi, A.J. Freeman, E. Wimmer, and M. Weinert, Phys. Rev. Lett. 50, 761 (1983).

18) M. Posternak, A. Baldereschi, A.J. Freeman, and E. Wimmer, Phys. Rev. Lett. $\mathbf{5 2 ,} 863$ (1984).

19) A. Catellani, M. Posternak, A. Baldereschi, and A.J. Freeman, Phys. Rev. B 36, 6105 (1987).

20) X. Blase, A. Rubio, S.G. Louie, and M.L. Cohen, Phys. Rev. B 51, 6868 (1995).

21) N.-T. Cuong, M. Otani, and S. Okada, J. Phys. Condens. Matter 26, 135001 (2014).

22) T. Enoki, M. Suzuki, and M. Endo, Graphite Intercalation Compounds and 
Applications (Oxford University Press, Oxford, 2003).

23) M. Otani and S. Okada, J. Phys. Soc. Jpn. 79, 073701 (2010).

24) M. Otani and S. Okada, Phys. Rev. B 83, 073405 (2011).

25) Q. Liu, Z. Li, and J. Yang, Chinese J. Chem. Phys. 24, 22 (2011).

26) A. Yamanaka and S. Okada, Appl. Phys. Express 7, 125103 (2014).

27) P. Hohenberg and W. Kohn, Phys. Rev. 136, B864 (1964).

28) W. Kohn and L.J. Sham, Phys. Rev. 140, A1133 (1965).

29) M. Otani and O. Sugino, Phys. Rev. B 73, 115407 (2006).

30) Y. Morikawa, K. Iwata, and K. Terakura, Appl. Surf. Sci. 169-170, 11 (2000).

31) J.P. Perdew and A. Zunger, Phys. Rev. B 23, 5048 (1981).

32) D.M. Ceperley and B.J. Alder, Phys. Rev. Lett. 45, 566 (1980).

33) D. Vanderbilt, Phys. Rev. B 41, 7892 (1990). 